\title{
Some consequences of including low frequencies in the evaluation of floor impact sound
}

\section{Rindel, Jens Holger; Rasmussen, Birgit}

\section{Published in:}

Acoustical Society of America. Journal

Link to article, DOI:

10.1121/1.416387

Publication date:

1996

\section{Document Version}

Publisher's PDF, also known as Version of record

Link back to DTU Orbit

\section{Citation (APA):}

Rindel, J. H., \& Rasmussen, B. (1996). Some consequences of including low frequencies in the evaluation of floor impact sound. Acoustical Society of America. Journal, 100(4, Pt. 2), 2769-2769.

https://doi.org/10.1121/1.416387

\section{General rights}

Copyright and moral rights for the publications made accessible in the public portal are retained by the authors and/or other copyright owners and it is a condition of accessing publications that users recognise and abide by the legal requirements associated with these rights.

- Users may download and print one copy of any publication from the public portal for the purpose of private study or research.

- You may not further distribute the material or use it for any profit-making activity or commercial gain

- You may freely distribute the URL identifying the publication in the public portal 


\title{
TECHNICAL NOTES AND RESEARCH BRIEFS
}

\author{
Paul B. Ostergaard \\ 10 Glenwood Way, West Caldwell, New Jersey 07006
}

Editor's Note: Original contributions to the Technical Notes and Research Briefs section are always welcome. Manuscripts should be double-spaced, and ordinarily not longer than about 1500 words. There are no publication charges, and consequently, no free reprints; however, reprints may be purchased at the usual prices.

\section{Error in the calculation of synchronized spontaneous otoacoustic emission frequencies measured with the ILO88 system [43.64.Jb, 43.64.Yp] \\ Jacek Smurzynski and Rudolf Probst}

Department of Otorhinolaryngology, University of Basel, Kantonsspital, CH-4031 Basel, Switzerland

High-level spontaneous otoacoustic emissions (SOAEs) were measured from 16 ears using both spectral and time averaging. The purpose was to determine the source of an upward shift in frequencies of synchronized SOAEs (SSOAEs) observed while using a subroutine of the ILO88 system of Otodynamics Ltd. An HP3561A signal analyzer performed spectral averaging to extract SOAEs with no external stimulation applied to the ear canal. Synchronized SOAEs were derived using the ILO88 system performing time averaging following click stimulation. The frequencies of all SSOAEs were shifted upwards by 6 to $21 \mathrm{~Hz}$ when compared to corresponding SOAE frequencies determined with spectral averaging. Additional measurements of signals in a cavity and of click-evoked otoacoustic emissions in selected ears indicated that the frequency shift is the result of an error in the ILO88 software. Incorrect cursor readouts in the program cause an apparent upward shift in frequency of $12.2 \mathrm{~Hz}$. This error was confirmed by the manufacturer.

Spontaneous otoacoustic emissions (SOAEs) represent narrow-band signals that can be recorded in the outer ear canal when no external acoustic stimulation is presented (see Probst et al., 1991 for a review). In general, two methods have been used to record them. In the first, the sound-pressure level in the ear canal is measured by a low-noise microphone with no stimulation applied. The microphone signal is averaged in the frequency domain (e.g., Whitehead et al., 1993). The second method consists of recording SOAEs synchronized by acoustic stimuli, for example clicks, using averaging in the time domain. This enables the detection of long lasting oscillations following click-evoked otoacoustic emissions (CEOAEs). It has been shown that for an ear with strong SOAEs, a CEOAE spectrum exhibits peaks corresponding to SOAE frequencies (Probst et al., 1986; Gobsch and Tietze, 1993). Software of a widely used commercially available instrument for measuring OAEs, the ILO88 (Otodynamics Ltd., Hatfield, UK), includes a subroutine for measuring synchronized spontaneous otoacoustic emissions (SSOAEs). Several recent studies have reported SSOAE data collected with the ILO88 system (Wable and Collet, 1994; Kulawiec and Orlando, 1995; Prieve and Falter, 1995). As part of an ongoing study of otoacoustic emissions in normal-hearing humans in our laboratory, we have measured SOAEs using both spectral averaging and the synchronization technique of the ILO88. In comparing the two results from the same ear, we have observed a slight but consistent difference in the frequencies of SSOAEs and SOAEs. Therefore, we sought to characterize this discrepancy further and to determine its source. Because of the widespread use of the ILO system, we believe that it is important that our findings be reported.

Both ears of eight subjects from our laboratory pool who had known SOAEs that were at least $10 \mathrm{~dB}$ above the noise floor of the instrumentation were tested with two methods. In the first method, the sound-pressure level in the ear canal was measured by a low-noise microphone (Etymotic Research, ER10A) with no stimulation applied. The microphone output was connected to a custom-made low-noise preamplifier with a gain of $20 \mathrm{~dB}$ and a high-pass filter with a cutoff frequency of $400 \mathrm{~Hz}$. The signal was led to a dynamic signal analyzer (Hewlett-Packard 3561A) and was analyzed from 0.5 to $10 \mathrm{kHz}$ in $500-\mathrm{Hz}$ Hanning windows. For each frequency span, a spectrum with an analysis bandwidth of $1.875 \mathrm{~Hz}$ was obtained based on the average of 20 fast Fourier transforms (FFTs) of the microphone signal.
Automatic artifact rejection of the spectrum analyzer with the rejection threshold set at $50 \mathrm{~dB}$ SPL was used to eliminate samples contaminated by high-level physiological noise resulting from swallowing, noisy breathing, and muscle and joint movements. Individual traces were stored in the bubble memory of the instrument for further analysis. A high-level SOAE was defined as any narrow-band signal that exceeded the noise floor by $\geqslant 10 \mathrm{~dB}$ and was present in two consecutive average spectra of the signal from the ear under test. The cursor on the display of the instrument was used to determine the frequency of the SOAE.

The second method used the SSOAE subroutine of version 4.2 of the ILO88 system's software run on a Pentium computer. In this mode, a single $80-\mu$ s pulse was presented every $80 \mathrm{~ms}$ at a peak level of approximately 72 dB SPL through a probe fitted in the external ear canal. The microphone signal was averaged over a 20 - to 80 -ms poststimulus time interval. Five hundred responses were averaged. The FFT resolution was approximately 12.2 Hz. Data were stored on a disk for further analysis. The characteristics of the SSOAEs were determined after examining two spectra, i.e., signal spectrum and noise spectrum, that were calculated and displayed by the ILO88 system. The frequency of the SSOAE corresponding to the maximum level of the narrow-band signal in the targeted frequency range was determined using a cursor. Testing using both methods was performed in a sound-treated room with the subject seated quietly. Each session started with SSOAE testing for both ears of one subject followed by SOAE testing.

The total number of high-level SOAEs ranged from 1 to 7 per ear (with the total number of 41 in 16 ears). There was generally good correspondence of the SOAEs and SSOAEs obtained from the same ears with the two methods of recording, i.e., a spectrum obtained with the ILO88 system exhibited peaks in the same frequency regions as detected by the HP3561A analyzer. Similar findings have been obtained previously by Gobsch and Tietze (1993) who used a laboratory system for data collection. Their Fig. 1, which depicts a representative example of SOAEs and SSOAEs, showed the presence of SSOAEs at exactly the same frequencies as SOAEs. A detailed analysis of our own data revealed, however, an evident shift between SSOAE and SOAE frequencies. For all 41 SOAEs, the corresponding SSOAE frequencies were higher by 6 to $21 \mathrm{~Hz}$. The observed difference between the frequencies is not due to differences in the frequency resolution of the two methods of recording $(1.875 \mathrm{~Hz}$ versus $12.2 \mathrm{~Hz})$ because in all cases the frequency shift was in the same direction. Therefore, we sought to determine the source of the error. One possible explanation for the differences might be that synchronizing clicks change the mechanical properties of the cochlea, which could result in a frequency shift. Interactions between SOAEs and external pure tones are known to exist (e.g., Long et al., 1991). Another possibility would be an artifact in the ILO system. Additional measurements were performed to evaluate these potential causes.

Figure 1 displays a set of results obtained from the left ear of one of the subjects. The data are for the $1750-$ to $2250-\mathrm{Hz}$ frequency region. The output recorded using spectral averaging with the HP3561 A analyzer is presented in Fig. 1(a). Note the dominant SOAE at $2035 \mathrm{~Hz}$ with a level of $+1.9 \mathrm{~dB}$ SPL (approximately $21 \mathrm{~dB}$ above the noise floor). Using the ILO88 system in SSOAE mode, a spectrum with several local peaks (SSOAEs), one of them at $2051 \mathrm{~Hz}$ with a level of $-8.1 \mathrm{~dB}$ SPL was obtained [Fig. 1(b)]. In this case, there was an upward shift of the SSOAE frequency by 16 $\mathrm{Hz}$ when compared to the SOAE frequency. Without removing the ILO88 probe from the ear canal and without changing the stimulus level, a CEOAE was recorded using the "nonlinear" operational mode (Kemp et al., 1990). This consists of a stimulus set of four clicks, three delivered at the same level and polarity and the fourth three times greater in level and inverted in polarity. The sweep time clock was set to $120 \mu$ s. The poststimulus analysis time window was $60 \mathrm{~ms}$, and the frequency resolution of the CEOAE spectrum was $16.3 \mathrm{~Hz}$. The CEOAE spectrum exhibited several local peaks with a strong narrow component at $2034 \mathrm{~Hz}$ [Fig. 1(c)], which corresponded 


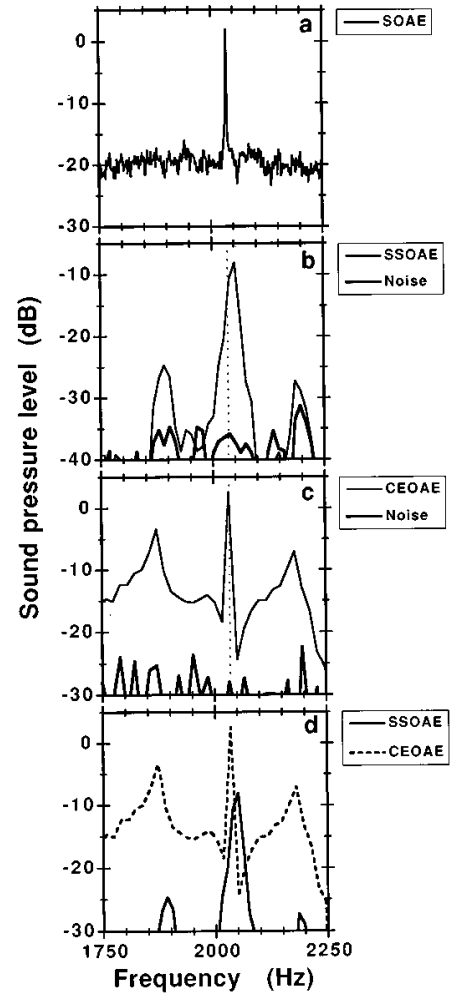

FIG. 1. Microphone signal spectra for a normally hearing ear collected using different methods of recording. (a) Spectral average $(n=20)$ obtained with the ER10A microphone and the Hewlett-Packard 3561A dynamic signal analyzer with an analysis bandwidth of $1.875 \mathrm{~Hz}$ depicts an SOAE at $2035 \mathrm{~Hz}$. (b) Time-averaged spectra obtained with the ILO88 system using the SSOAE search mode with a $12.2-\mathrm{Hz}$ frequency resolution. The response spectrum (thin line) depicts an SSOAE at $2051 \mathrm{~Hz}$ clearly detectable above the background noise (thick line). (c) Time-averaged spectra obtained with the ILO88 system using the nonlinear CEOAE mode and a $16.3-\mathrm{Hz}$ frequency resolution. The response spectrum (thin line) depicts a strong narrow component at $2034 \mathrm{~Hz}$. Thick line represents background noise. The vertical dotted line in panels (b) and (c) corresponds to the frequency of the SOAE at $2035 \mathrm{~Hz}$ determined by the spectrum analyzer. (d) The SSOAE spectrum (solid line) overlaid on the CEOAE spectrum (dashed line).

almost exactly to the frequency of the SOAE determined using spectral averaging [Fig. 1(a). Note: The difference of $1 \mathrm{~Hz}$ is lower than the frequency bandwidth of the spectrum analyzer.] A comparison of the two spectra collected with the ILO88 system in the two different operational modes showed an obvious difference [Fig. 1(d)]. Similar results were obtained from four other ears. This finding does not support the hypothesis that synchronizing clicks might create a "physiological" shift of the SOAE frequency because both the SSOAE and CEOAE modes of the ILO88 system use click stimulation. Therefore, we sought to determine if the observed shift between SOAE and SSOAE frequencies might be an artifact.

For these tests, an SES coupler (Keller and Pedersen, 1987) was used instead of a human ear. An Etymotic Research ER-3 earphone was inserted into the coupler to deliver a pure tone generated by a synthesizer. The frequency was $2035 \mathrm{~Hz}$, which represents the SOAE from the left ear of the subject whose results are in Fig. 1. The ER10A microphone was also sealed into the cavity, and the signal spectrum was measured using the same method of spectral averaging as used for the SOAE testing. The frequency of the pure tone in the spectrum recorded from the cavity matched the SOAE from the human ear as displayed in Fig. 1(a). The ER10A microphone was removed and the ILO88 probe was then sealed into the cavity. The spectrum collected with the SSOAE mode of the ILO88 system exhibited a peak at $2051 \mathrm{~Hz}$, which was the SSOAE frequency exhibited by the subject [Fig. 1(b)]. Thus, the data collected from the cavity support the view that the upward frequency shift observed in the SSOAEs collected with the ILO88 system is an artifact.

Figure 1(b)-(d) was generated by accessing original ILO88 data files and creating new text files representing response and noise spectra. These new files were checked carefully to determine if values corresponding to response and noise levels at a particular frequency were the same as displayed on the computer monitor when a cursor was moved with a step size of 12.2 or $16.3 \mathrm{~Hz}$ for the SSOAE or CEOAE mode, respectively. No discrepancy was found. This pointed to an error in the cursor readout of the SSOAE subroutine of the ILO88. A file created during the SSOAE test would be read incorrectly when results are displayed on the screen. Namely, the frequency depicted by the cursor would be shifted upwards in relation to its actual value. An inquiry to the manufacturer of the equipment (Otodynamics Ltd.) led them to examine the source code of their software, and the error was detected and confirmed. The leftmost position of the cursor was set incorrectly to $12.2 \mathrm{~Hz}$ rather than to zero as it should have been. This accounts for the $12.2-\mathrm{Hz}$ frequency shift of the cursor readout. After correcting for this error, the difference between the frequencies of the 41 SOAEs and SSOAEs recorded from the group of 16 ears would range from -6 to $+9 \mathrm{~Hz}$.

The data shown in Fig. 1 illustrate additional findings (currently under further exploration) that are outside the main focus of this study but need some clarification in this paper. For all 16 ears, the SOAEs exhibited higher levels than corresponding SSOAEs. For example, there was a 10-dB difference in the level of the SOAE shown in Fig. 1(a) compared to its SSOAE counterpart shown in Fig. 1(b). The reason of that difference is somewhat unclear. A set of SOAE and SSOAE recordings is planned using the same ER10A probe and our own software to control data acquisition via a 16-bit A/D converter for both methods. These measurements would also allow a direct comparison of S/N for SOAE and SSOAE recordings. Such a comparison based on the present data is difficult due to unknown characteristics of the ILO88 probe. Finally, in almost all of the 16 ears, the spectra obtained with the ILO88 system exhibited additional peaks (SSOAEs) that were not observed on the spectra recorded with the HP3561A analyzer (e.g., Fig. 1). It is believed that those peaks represented synchronized sound-evoked components with a long duration but without spontaneous oscillations. Similar findings were previously reported by Wable and Collet (1994).

In conclusion, the ILO88 system, which is commercially available and widely used to measure CEOAEs in clinical settings, is also valuable for screening for the presence of SSOAEs. For such screening purposes, the frequency discrepancy of $12.2 \mathrm{~Hz}$ is not of critical importance. However, when detailed information regarding SOAEs is required SSOAE data should be corrected accordingly when version 4.20 (or earlier) of the software is used. Frequencies of SSOAEs are incorrectly displayed on the computer screen and should be corrected by shifting frequency downward by one step, i.e., $12.2 \mathrm{~Hz}$.

\section{ACKNOWLEDGMENTS}

This study was supported by a grant from Swiss National Foundation (project Nr. 3200-042241.94/1). We thank Dr. Dennis McFadden for stimulating discussions via the Internet, Dr. David Kemp for helping us to solve the "mystery" of the frequency shift, and Dr. Frances Harris for comments on the manuscript.

Gobsch, H., and Tietze, G. (1993). "Interrelation of spontaneous and evoked otoacoustic emissions," Hear. Res. 69, 176-181.

Keller, F., and Pedersen, P. (1987). "Der simulierte Ear Simulator (S.E.S.),' Z. Hörgeräte-Akustiker 5, 6-16.

Kemp, D. T., Ryan, S., and Bray, P. (1990). "A guide to the effective use of otoacoustic emissions," Ear Hear. 11, 93-105.

Kulawiec, J. T., and Orlando, M. S. (1995). "The contribution of spontaneous otoacoustic emissions to the click evoked otoacoustic emissions," Ear Hear. 16, 515-520.

Long, G. R., Tubis, A., and Jones, K. L. (1991). “Modeling synchronization and suppression of spontaneous otoacoustic emissions using Van der Pol oscillators: Effects of aspirin administration," J. Acoust. Soc. Am. 89, $1201-1212$.

Prieve, B. A., and Falter, S. R. (1995). "COAEs and SSOAEs in adults with increased age,"' Ear Hear. 16, 521-528. 
Probst, R., Coats, A. C., Martin, G. K., and Lonsbury-Martin, B. L. (1986). "Spontaneous, click-, and toneburst-evoked otoacoustic emissions from normal ears,' Hear. Res. 21, 261-275.

Probst, R., Lonsbury-Martin, B. L., and Martin, G. K. (1991). “A review of otoacoustic emissions,', J. Acoust. Soc. Am. 89, 2027-2067.
Wable, J., and Collet, L. (1994). "Can synchronized otoacoustic emissions really be attributed to SOAEs?,' Hear. Res. 80, 141-145.

Whitehead, M. L., Kamal, N., Lonsbury-Martin, B. L., and Martin, G. K. (1993). "Spontaneous otoacoustic emissions in different racial groups," Scand. Audiol. 22, 3-10.

\section{Advanced-degree dissertations in acoustics}

Editor's note: Abstracts of Doctoral and Master's theses will be welcomed at all times. Please note that they must be double spaced, limited to 200 words, must include the appropriate PACS classification numbers, and formatted as shown below (don't make the editor retype them, please!). The address for obtaining a copy of the thesis is helpful. Please submit two copies.

Multiple-reference adaptive noise control in enclosures[43.40.Qi, 43.50.Ki, 43.60.Lq]-Zane Michael Rhea, Graduate Program in Acoustics, The Pennsylvania State University, University Park, PA 16802, May 1996 (M.S.). Noise control of multiple noise sources in threedimensional enclosures remains a concern in many applications. This is of particular interest in vehicles, such as twin-engine propeller-driven aircraft, where multiple primary sources contribute to the sound field inside the enclosure. When utilizing active noise control (ANC) systems in these applications, multiple reference inputs are required. A comparison of ANC of multiple noise sources with multiple and single reference inputs is presented in a case format. The enclosure walls were excited at four pairs of frequency sets: (1) with both frequencies on-resonance for both the structure and the enclosure, (2) with both frequencies at structurally dominated modal frequencies (on-structural off-enclosure resonance), (3) with both frequencies at acoustically dominated modal frequencies (off-structural on-enclosure resonance), and (4) with both frequencies off-resonance for both the structure and enclosure. The purpose of this study is to determine if the enclosed sound field from multiple noise sources can successfully be controlled with (1) a single reference input which contains information from only one primary noise source, (2) a single reference input which contains information from both primary sources, and (3) multiple reference inputs; at frequencies on and off resonance for the structure and enclosed space.

Thesis co-advisors: Courtney B. Burroughs, Scott D. Sommerfeldt.

Remote estimation of sound speed in an elastic medium [43.30.Es, 43.30.Pc, 43.58.Dj]—Todd A. Matthias, Graduate Program in Acoustics, The Pennsylvania State University, University Park, PA 16802, December 1995 (M.S.). Remotely determining the acoustic properties of an unknown medium such as the ocean sediment is a fundamental problem of interest to ocean acousticians. This thesis describes several nondestructive, remote methods for finding the sound speed in a medium that is adjacent to a medium of known sound speed. The methods are developed and examined for accuracy. An experiment is performed in which one of these methods is used to determine the sound speed in an elastic medium submerged in water.

Thesis advisor: R. Lee Culver.

Active control of discrete-frequency noise from small subsonic fans[43.50.Ed, 43.50.Ki, 43.28.Ra]-John MacGillivray, Graduate Program in Acoustics, The Pennsylvania State University, University Park, PA 16802, May 1996 (M.S.). This research investigates the use of active noise control to reduce the emissions of small subsonic axial flow fan units typically found in computer enclosures and printers. Aerodynamic noise generated by an axial flow fan is reduced using active noise control where sources of tonal sound in the primary noise field are coupled to a secondary source created by physically shaking the fan unit. The nature of the control method essentially colocates the primary and secondary sources at frequencies for which the fan acts as a compact source. Feasibility studies are used to predict potential fan and shaker combinations that would be effective and to determine which implementations of control are viable. Simulations of the proposed control scheme are presented and an experimental demonstration of the concept using a 92-mm axial flow fan which is baffled is con- ducted. Sound power level reduction of $14 \mathrm{~dB}$ at the blade passage frequency of a baffled fan unit is achieved, and additional measurements show that sound pressure level reductions as great as $21 \mathrm{~dB}$ can be demonstrated for a fan surrounded by a computer enclosure.

Thesis advisor: Dr. G. C. Lauchle.

A modal analysis technique for observing precursors to mechanical failure[43.40.Le, 43.40.Qi]—Douglas P. Koehn, Graduate Program in Acoustics, The Pennsylvania State University, University Park, PA 16802, May 1996 (M.S.). An experiment is developed to measure the resonant responses during fatigue of a steel band. Broadband vibrations are induced in the band by means of an electromechanical shaker in order to fatigue the sample until failure. The frequency response is measured at various times throughout this process over a range of frequencies. A simple mathematical model shows the effects on the resonance frequencies as a function of the changes in the band's stiffness and area, representing the effects of a crack in the band. Tracking frequencies of resonance and amplitudes of the resonant responses throughout the fatigue cycle show varying results among samples and various times until material failure. Though the results vary, comparing the results with a reference samples suggests that the changes in the frequencies of resonance are due to changes in material properties and crack effects rather than random error in the experimental procedure. In general, it appears that frequencies of resonance tend to decrease near the point of structural failure as cracks grow and material stiffness decreases. More sophisticated automated data collection techniques are proposed for gathering data which may lead to a prognosis of material failure.

Thesis advisor: David C. Swanson.

Calibration and validation of an interfacial acoustic intensity probe[43.20. Tb, 43.20.Ye, 43.40.Rj, 43.58.Fm]-Paul C. Janzen, Graduate Program in Acoustics, The Pennsylvania State University, University Park, PA 16802, December 1995 (M.S.). Sound intensity has been traditionally measured using two closely spaced microphones to measure the acoustic pressure and particle velocity. This method is widely used, but the equipment is expensive and the phase responses of the microphones and the measurement equipment must be precisely matched. This thesis describes an alternate way to measure sound intensity which operates at the interface between a vibrating structure and the fluid. The interfacial intensity probe (IIP) consists of a microphone and an accelerometer mounted in a single package, which is attached to a radiating structure. The microphone measures the pressure, and the accelerometer measures the normal acoustic particle velocity at the interface between the structure and the fluid. The IIP is much less costly than a typical two-microphone probe, and the phase requirements for the instrumentation are much less strict. An overview of traditional intensity measurement techniques is followed by descriptions of the probe itself, experiments performed to analyze the performance of the probe, and the errors involved in making sound power measurements using an IIP. The experiments showed that the IIP can indeed accurately measure the sound power transmitted through a flat plate.

Thesis advisor: Gary H. Koopmann.

Gender differences and the development of the frequency importance functions using the articulation index[43.71.Bp, 43.71.Es, 43.71.Gv]-Mary Colleen Herr, Graduate Program in Acoustics, The Pennsylvania State University, University Park, PA 16802, December 1995 (M.S.). Since the early study by French and Steinberg (1947), there has been little female data involved in frequency importance function calculations. This research investigated the frequency importance function developed for words spoken by male and female speakers. The 
highly controlled experimental conditions ensured that differences in FIF were attribute to gender alone. Twenty-two normal hearing listeners were asked to identify words presented under 60 filtering and noise background conditions for each gender of speaker. The frequency importance functions for words spoken by both speakers were derived and graphically presented. The most important frequency band for understanding male and female produced speech, was in the $1400-$ to $3000-\mathrm{Hz}$ frequency band. A statistical analysis on the differences between the FIFs was also conducted using a one-way ANOVA and one-sample runs test. The statistical analysis found that there was no significant difference between the frequency importance functions for words spoken by the two speakers. It was concluded that gender did not play a role as a distinguishing characteristic in frequency importance functions for words spoken by the particular set of speakers participating in this study. Future studies may include the utilization of child speakers to obtain frequency importance function information for various speech types.

Thesis advisor: Claus P. Janota. A superposition model for the study of acoustic radiation by
finite length cylinders[43.20. $\mathrm{Bi}, 43.20 . \mathrm{Rz}, 43.30 . \mathrm{Jx}, 43.40 . \mathrm{Ey}]-$
Mark J. Bregar,Graduate Program in Acoustics, The Pennsylvania State University, University Park, PA 16802, May 1995 (M.S.).Cylinders, one of the fundamental geometric shapes, are found in many physical devices. In applied engineering and acoustics, the study of vibrating cylinders becomes important in structural analysis and noise control. Cylinder modeling, therefore, becomes an important tool in determining their behavior. Exact solutions to their acoustic field patterns become unmanageable due to the mathematic complexities. Boundary element models are available commercially, but,a simple model that can be run on a small digital computer is desired. The superposition principle arises as a plausible candidate. In order to confirm its appropriateness and discover the limitations of the model, a study was undertaken. The following five steps were taken: (1) formulate and justify the model mathematically; (2) prove, by a comparison of two dimensional exact and superposition model solutions, that indeed the representation provides acceptable results; (3) extend the model to the more practical three dimensions; (4) apply it to "real world" problems and (5) compare the results to available empirical data. A review is then made which exhibits the strengths and weakpoints of the superposition formulation.

Thesis advisor: Oliver McDaniel. 